\title{
Clustering of Proteins Embedded in Lipid Bilayers: a Monte Carlo Study
}

\author{
Marianna Yiannourakou ${ }^{*+}$, Luca Marsella ${ }^{*}$, Frédérick de Meyer ${ }^{*}$ and Berend Smit ${ }^{*}$ \\ ${ }^{*}$ Centre Europeén de Calcul Atomique et Moleculaire, 46 Allée d'Italie 69364 Lyon, France \\ ${ }^{+}$Molecular Thermodynamics and Modeling of Materials Laboratory, Institute of Physical Chemistry, \\ National Center for Scientific Research “Demokritos”, GR-153 10, Aghia Paraskevi Attikis, Greece
}

\begin{abstract}
The clustering behavior of peptides embedded in a lipid bilayer is extensively studied using a two-dimensional coarse-grained model under different conditions of hydrophobic mismatch. We observe the formation of stable clusters of peptides with size exhibiting an upper limit. This result accounts for a natural tendency of peptides to aggregate, responding to the perturbation induced in the membrane, confirming previous experimental and computational findings.
\end{abstract}

Keywords: Membrane, bilayer, proteins, peptides, lipids, hydrophobic mismatch, clusters

PACS: 87.14.Cc, 87.14Ee, 87.15.Aa

\section{INTRODUCTION}

The study of biological membranes has drawn much attention during the past years, in the effort to relate their physical properties to the regulatory processes of vital importance for cell life that they accomplish. The difficulty of studying these systems is twofold: first, membranes are complex cooperative structures where a variety of biological molecules may be embedded. Furthermore, one needs to take into account a broad range of sizes and timescales for the processes occurring within them.

In order to overcome these difficulties, it is essential to study reconstituted systems, which model simple membranes. Moreover, different models may be used to treat these systems at different levels of detail, in order to cope with the different timescales involved in the process of interest.

Lipid bilayers composed of one or two lipid species, together with embedded proteins, provide a good model for membranes: recently, effective interactions between peptides embedded in membranes have been widely studied adopting computational approaches, both with mesoscopic modeling and atomistic simulations ${ }^{1,2}$. Peptides insertion perturb the local structure of the membrane, resulting in long range interactions between the embedded molecules, which can explain the clustering of transmembrane peptides and can be followed for a time of up to hundreds of nanoseconds using atomistic molecular dynamics simulations ${ }^{2}$.

However, due to the large time and length scales involved in peptide clustering within the membrane systems, such studies need to be limited to few peptides and model membranes of small sizes. Coarse-graining techniques allow to bridge the gap between atomistic and phenomenological description and can thus be used for an integrated study on the activity of transmembrane peptides, retaining the notion of molecular conformations and qualitatively capturing the properties of the local environment. The recently developed technique of Dissipative Particle Dynamics (DPD) and the use of coarse graining techniques allow to investigate the collective behavior of peptides inside the lipid membrane on longer time scales.

Depending on the level of the coarse-graining, there is a trade-off between the information obtained by the simulation and the ability to study larger systems for longer times. Venturoli and coworkers ${ }^{2}$ have used a coarsegraining model of a lipid bilayer with embedded proteins under DPD, where lipids are represented as a chain of soft beads that can be either hydrophilic (head) or hydrophobic (tails) (Fig.1). Using the techniques developed for determining the effective interactions between colloids, one can obtain the parameters for such a coarse-grained description from small all-atom simulations. Moreover, the effect of hydrophobic mismatch ${ }^{3}$ (the difference of the 
hydrophobic lengths of the protein and the lipids) has been explored. The results obtained from these mesoscopic simulations are in qualitative good agreement with previous theoretical predictions for the dependence on mismatch and protein size of the extent of the perturbation caused by the protein on the nearby lipids, as well as with the few experimental quantitative estimates of the range of the perturbation that some specific proteins (such as bacterio rhodopsin, lactose permease and synthetic alpha-helical peptides) induce on reconstituted pure lipid bilayers.
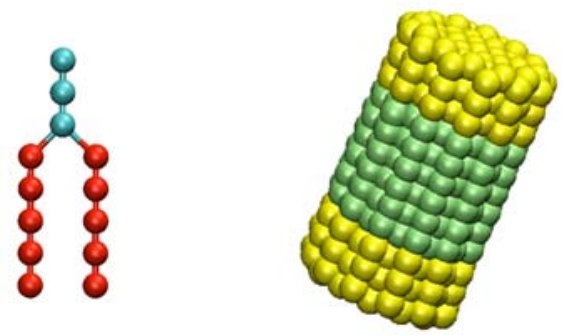

FIGURE 1 Left: Schematic representation of model lipids, the blue particles represent the hydrophilic head-beads and the red particles the hydrophobic tail-beads, and Right: protein

Such an agreement between the model and the data from previous theoretical or experimental studies confirms the reliability of the approach based on the DPD simulation method applied at mesoscopic scales.
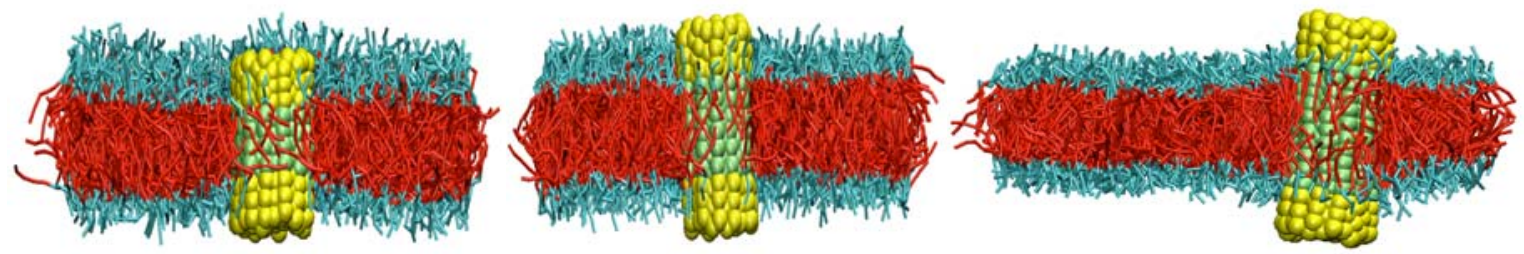

FIGURE 2 Lipid bilayer with an embedded peptide. Left: negative mismatch, lipid tails around the peptide shorten their hydrophobic length to avoid the hydrophilic head of the protein, Centre: zero mismatch, no rearrangement is needed, Right: positive mismatch, lipid tails are stretched around the protein to shield its hydrophobic side from water.

Although such a mesoscopic model reproduces some properties of real systems, simulations of big systems with many embedded proteins for long timescales are computationally prohibitive, due to the large number of degrees of freedom involved.

In the present work we have moved to a higher level of coarse-graining, in order to study larger systems,. The model that we have used will be described in the following section. Our aim is to study big systems of lipid bilayers with a large number of embedded proteins. In particular, we focus on proteins clustering behavior for each case of mismatch (negative, zero and positive). As input, we used the information obtained by simulations of the mesoscopic model mentioned above.

\section{MODEL AND SIMULATION METHOD}

De Meyer and coworkers ${ }^{4}$ have calculated the potential of mean force (PMF) through which embedded peptides of different sizes and hydrophobic mismatch effectively interact, due to the mediation of the lipid bilayer (Fig.2).

Our study focuses on systems of proteins that approximately have the size of gramicidin and are embedded in a dimyristoylphosphatidylcholine (DMPC) bilayer. The PMFs of these systems corresponding to negative and positive hydrophobic mismatch (Fig.3) show a very intriguing feature: for small distances between the two proteins, their interaction is attractive, while at larger distance, they show a small repulsion; for relatively larger distances, the two proteins do not feel each other. The presence of the attractive well in the PMF of two interacting proteins accounts for the tendency of embedded peptides to form clusters, up to a limiting size characteristic of the system, which is controlled by the position and height of the repulsive barrier. Indeed, clustering of proteins and formation of protein aggregates have been observed both experimentally and in computer simulations $1,5,6,7,8$.

In order to study the clustering properties of this model system, we adopt an even simpler description of the process, switching to a two-dimensional model of the bilayer. 
This higher level of coarse-graining employs a two dimensional simulation box in which proteins are modeled as hard disks interacting via the PMFs of Fig.3, depending on the type of hydrophobic mismatch. The lipid medium disappears, since its presence is taken into account implicitly by the PMF.
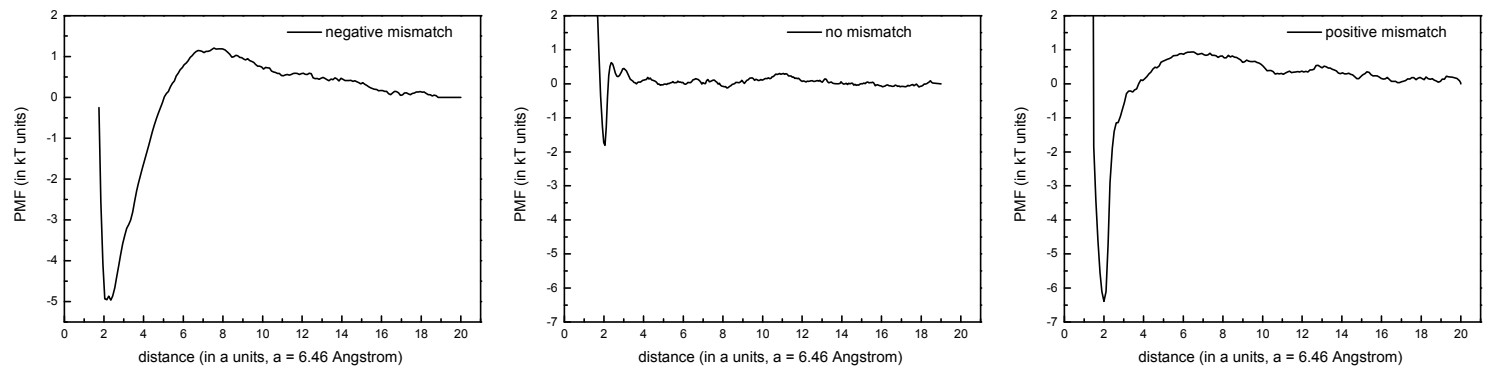

FIGURE 3 For each case of hydrophobic mismatch, coarse grained simulations are conducted and the PMF (Potential of Mean Force) is being computed. (a) Negative, (b) zero and (c) positive mismatch.

We are thus able to simulate larger systems with cluster formation and to compute the associated free-energy as a function of the cluster size. We can have an estimation on the clustering behavior of proteins embedded in the lipid bilayer depending on the type of the mismatch. Of course the level of the coarse-grained approach doesn't allow the study of inherently three dimensional details of the clusters, such as tilting of the peptides or local deformation of the membrane thickness. However this exceeds the scope of this study: our findings may be further used as guidelines to design three dimensional simulations or experiments that may look into the details of the clustering phenomena.

We are neglecting on purpose three-body and higher order interactions among embedded proteins, to keep the description at the simplest level: the density of the system is kept low $(<0.0025$ particles per unit surface $)$ in order to reduce the relevance of higher order interactions and be able to use the PMF as a pairwise potential.

We perform Monte Carlo (MC) simulations in the NVT ensemble, adopting cubic splines to interpolate the numerical PMF obtained by coarse-grained MD simulations. Following Sperotto and coworkers ${ }^{9}$, we use as well a more regular fit with transcendental functions, whose parameters might be related to some system properties, such as the hydrophobic mismatch and the characteristic length of the perturbation induced by peptide insertion in the bilayer. Such a fit allows the application of conjugate gradient methods ${ }^{10}$ to compute ground states energy and the corresponding configurations as a function of cluster sizes.

The initial configuration of each run is such that proteins are placed at a distance larger than the cut-off radius $\left(\mathrm{R}_{\text {cut }}\right)$. We follow the behavior of a system with $\mathrm{N}=400$ proteins, in order to study the clustering process. At every $\mathrm{MC}$ cycle, $\mathrm{N}$ translational single protein move and one cluster move attempts are performed. Single peptides or clusters are selected and displaced randomly within a fixed range. Splitting or joining of different clusters is forbidden. MC Moves are accepted or rejected according to the Metropolis algorithm. Linked Cell Lists are being used to speed the calculations. Even using cluster moves, the energy of the system as a function of MC cycles equilibrates slowly: the reason is that in order for the clusters to get bigger, smaller clusters will have to break up or at least allow proteins to escape. Therefore, the runs length is of the order of $10^{7} \mathrm{MC}$ cycles.

\section{RESULTS AND DISCUSSION}

A series of runs has been performed for each case of mismatch (negative, zero and positive): no clustering is observed for zero mismatch, so we will only discuss simulations on systems with negative and positive mismatch. By means of conjugate gradient, we were able to obtain the grand state energy of clusters of size up to 20 particles. The energy of the system drops monotonically as the size of the cluster is increased up to $n \approx 14$. The energy reaches a plateau for $\mathrm{n}=14-16$ and then it begins to rise again. This information guides our simulations and gives us an idea of the cluster sizes that may be formed. Of course, since our Monte Carlo simulations are being conducted at a reduced temperature of $\mathrm{T}=0.7$, we do not necessarily expect to see the formation of 14-protein size clusters.

Collecting data from MC runs at constant temperature, we were able to obtain the free energies of the clusters as a function of their size (Fig. 4) for negative and positive mismatch. 

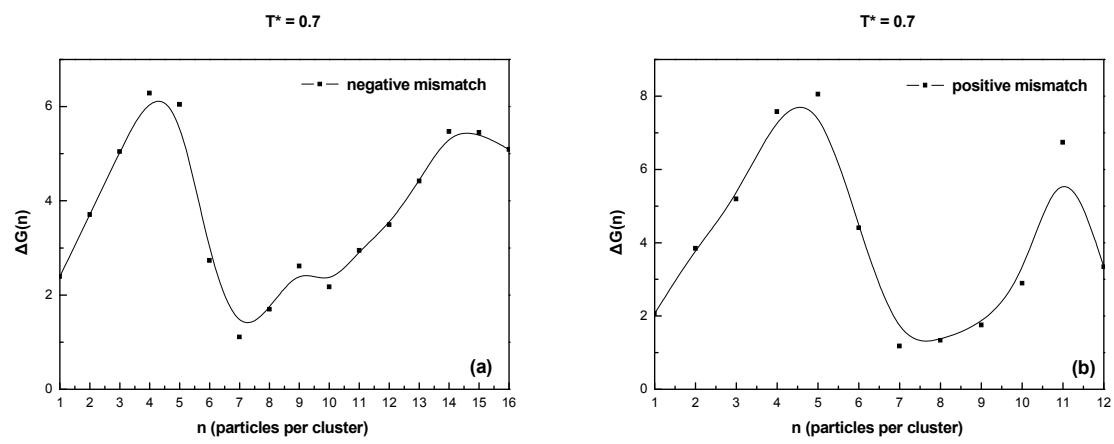

FIGURE 4 Free Energy differences for: (a) negative mismatch and, (b) positive mismatch systems.

For the case of negative mismatch, we can see that clusters of up to 16 particles are being formed. Clusters of 7 particles have the lowest free energy and are most probable to be formed. Two more free minimums are seen for clusters of 10 particles as well as for single particles. For the case of positive mismatch, the picture is almost the same. Clusters of up to 12 particles are being formed. Clusters of 7 to 9 particles as well as single particles have the lowest free energy and are most probable to be formed.

\section{CONCLUSIONS}

Using a two-dimensional representation of lipid bilayers with embedded proteins for the cases of negative and positive hydrophobic mismatch, we were able to observe the clustering behavior of peptides in the low density limit. Cluster formation takes place in both cases, with the clusters size exhibiting an upper limit. While the clusters size distribution depends strongly on the height of the repulsive barrier of the potential and on its distance from the centre of mass of the protein, the stability of the clusters is a qualitative result of this study that supports the tendency of proteins to aggregate when embedded in bilayers. This natural tendency of peptides to build bigger compounds tries to minimize the perturbation produced in the membrane, which is mainly due to the hydrophobic mismatch and the subsequent exposure of hydrophobic moieties to the polar solvent. Our results might be used as a guide for new simulations and experiments that will look into the conformational properties of clusters and the effects that their formation might produce on the bilayer.

\section{ACKNOWLEDGMENTS}

The authors would like to thank Maddalena Venturoli for numerous stimulating discussions and her help on both technical as well as theoretical aspects of this work. This work was partly supported by the EC through the Marie Curie projects: BiMaMoSi (MEXT-CT-2005-023311) and EuroSim (MEST-CT-2005-020491).

\section{REFERENCES}

H.Leontiadou, A.E.Mark, S.J.Marrink, J.Am.Chem.Soc. (2006) 128, 12156-12161.

M.Venturoli, B.Smit and M.M.Sperotto, Biophysical Journal (2005) 88, 1778-1798.

O.G. Mouritsen, M. Blom, Biophysical Journal (1984) 46, 141-153

F. de Meyer, M.Venturoli and B.Smit (to be submitted)

S. Morein, J.A. Killian, M.M. Sperotto, Biophysical Journal (2002) 82, 1405-1417

M. Diociaiuti, F. Bordi, A. Motta, A. Carosi, A. Molinari, G. Arancia, C. Coluzza, Biophysical Journal (2002) 82, 31983206

7 V.P. Ivanova, I.M. Makarov, T.E. Schäffer, T. Heimburg, Biophysical Journal, (2003) 84, 2427-2439

8 T. Gil, J. H. Ipsen, O.G. Mouritsen, M.C. Sabra, M.M. Sperotto, M.J. Zuckermann, Biochimica et Biophysica Acta (1998) $1376,245-266$

9 M.M.Sperotto, S.May and A.Baumgaertner, Chemistry and Physics of Lipids (2006) 141, 2-29

10 W.H.Press, S.A.Teukosky, W.T. Vetterling, B.P. Flannery, Numerical Recipes in Fortran 77 (2001) Cambridge University Press: Cambridge U.K. 